\title{
CONSTRUÇÃO DE PROTÓTIPOS POR ADIÇÃO E REMOÇÃO DE MATERIAL COMPARATIVO ENTRE DEPOSIÇÃO EM ABS X USINAGEM CNC
}

Alexandre de Aviz ${ }^{1}$

Cesar Augusto Guerra²

Tiago Carlos Guerra ${ }^{3}$

\begin{abstract}
RESUMO
Este artigo foi desenvolvido para traçar um comparativo na confecção de protótipos com duas diferentes tecnologias de fabricação: deposição em ABS e usinagem CNC. Nos últimos anos a prototipagem vem crescendo em grande escala, com o desenvolvimento de novas tecnologias focando o atendimento às necessidades demandadas pelo setor metalmecânico. Com o desenvolvimento destas tecnologias, os clientes ganharam uma gama de opções para o desenvolvimento de seus modelos prototipados. Com o auxílio de dois estudos de caso, foi possível identificar e analisar as principais características dos dois processos de fabricação de protótipos, deposição e remoção de material, ambos utilizando como matéria-prima o $A B S$, identificando suas vantagens e desvantagens em ambos os casos. Comparou-se duas peças com geometria pré-definida, onde, no primeiro caso (deposição de material), foi possível identificar problemas relacionados com o acabamento da superfície do protótipo, enquanto no segundo caso (usinagem CNC), identificou-se os resultados relacionados com a resistência mecânica, após os ensaios de tração realizados em uma série de corpos de prova dentro dos laboratórios do SENAI de Joinville, pelos técnicos laboratoriais. Realizando o comparativo com levantamento de custos, o processo de deposição teve um menor custo para peças menores, mas com o aumento de massa, o processo de usinagem CNC obteve um custo menor, devido, principalmente, ao tempo e material gasto.
\end{abstract}

Palavras chave: Prototipagem. Usinagem CNC. Deposição de Material.

\footnotetext{
${ }^{1}$ Especialista, e-mail: alexandre.aviz@sc.senai.br

2Especialista, e-mail: cimacad@cimacad.com.br

${ }^{3}$ Graduado Tecnólogo, e-mail: tiago.guerra@sc.senai.br
} 


\section{INTRODUÇÃO}

O sucesso no lançamento de um novo produto está associado diretamente à identificação de erros em sua fase inicial de desenvolvimento de produtos dentro da empresa e as suas habilidades em identificar as necessidades do cliente e reverter em soluções para os mesmos. Desta forma, a utilização de protótipos nestas etapas, pode auxiliar na identificação de erros antes do início da fabricação do produto final, como por exemplo, um protótipo de para-choque de caminhão que custa em média $2 \%$ do valor de uma ferramenta que sem o protótipo não é possível identificar erros de projeto que poderão afetar diretamente a qualidade do produto e seu prazo de lançamento.

A prototipagem dentro da área metal mecânica e desenvolvimento de novos produtos, auxilia na transformação de ideias de projeto em modelos "vivos", proporcionando uma maior assertividade na construção dos moldes.

Nos últimos anos o conceito de prototipagem e sua utilização dentro das empresas do setor Metal mecânico cresceram muito, possibilitando um maior e melhor atendimento às necessidades dos clientes envolvidos. Muitas vezes a fase de desenvolvimento de produtos esbarra na necessidade de "ideias tangíveis" para que consiga dar sequência em seu trabalho. Partindo desta ideia, este artigo vem como auxilio para a escolha mais prática de construção dos protótipos, visando a diminuição do lead time.

Dessa forma, será feita uma análise de dois processos de confecção de protótipos, um representando a prototipagem por adição de material, que é a deposição em ABS e outro por remoção de material, partindo de um bloco bruto e confeccionando em máquina CNC.

O presente artigo apresenta como objetivo geral desenvolver um estudo comparativo entre o processo de deposição de material e o de usinagem CNC na confecção de protótipos. E seus objetivos específicos, são eles: compreender os principais fatores relevantes envolvidos nos processos, de design/criação e prototipagem; entender os conceitos e etapas do processo e principais aplicações por meio de um levantamento de informações providas do estudo de caso; conhecer as diferentes técnicas de prototipagem por deposição de material e usinagem CNC; conhecer e identificar as vantagens em se utilizar protótipos nos desenvolvimento de novos produtos.

\section{$2 \quad$ PROTÓTIPOS}

Segundo Baxter (1998), o volume de investimento em um projeto de produto é sempre menor nas fases iniciais do desenvolvimento do que quando na fase inicial deste produto na economia de escala, segundo ele o protótipo vem primeiro em uma série de produtos. 
Os protótipos são peças físicas confeccionadas para auxiliar no desenvolvimento de novos produtos submetidos a testes e ensaios, antes de sua aprovação para confecção de ferramentas e moldes dos produtos, ou apenas para visualização de geometrias, onde é possível transferir a ideia para um "modelo vivo". De modo geral, uma das principais funções do protótipo é a aprovação final do produto, antes da sua produção definitiva.

Com o surgimento de novas tecnologias de prototipagem, o que era tarefa especializada e demorada tornou-se mais fácil, gerando um considerável aumento no uso de protótipos físicos. Além disso, abordagens como a da engenharia simultânea têm sido bastante facilitadas pelo uso de protótipos.

Para melhor entender a importância da prototipagem no processo de desenvolvimento de produtos, é fundamental visualizar o papel dos protótipos neste processo, para tanto, são necessários alguns comentários sobre o seu uso e vantagens, antes de entrar em mais detalhes sobre essas tecnologias.

O mercado encontra-se atualmente em um ambiente cada vez mais globalizado, onde o sucesso de uma empresa depende de que o desenvolvimento de seus produtos seja feito dentro das qualificações que o consumidor exige, em um tempo menor que seus concorrentes e essencialmente buscando tendências inovadoras, porém sempre respeitando o conceito de autossustentabilidade no meio em que a organização encontra-se inserida (LIMA, 2009).

O sucesso de um produto está muitas vezes associado à habilidade das empresas em identificar as necessidades dos clientes e imediatamente desenvolver os produtos que preencham as lacunas a um baixo custo. A aprendizagem rápida dos envolvidos em cada estágio do processo de desenvolvimento de um produto é a chave para o sucesso dele sendo a utilização de protótipos, quando devidamente explorada uma parte essencial no processo.

\subsection{A finalidade dos protótipos no desenvolvimento de produtos}

Com a necessidade de se atingir o mercado consumidor, que deseja formas mais harmoniosas, ou mesmo na fabricação de itens para as indústrias que utilizam um alto grau de complexidade geométrica em seus produtos, como por exemplo, a indústria aeronáutica, automotiva ou de moldes e matrizes, um intensivo avanço tecnológico nos processos de desenvolvimento de produtos vem acontecendo, visando manter a qualidade exigida e ao mesmo tempo obtendo um ciclo de desenvolvimento de produto reduzido (TOLEDO; BRITO, 1999).

Os protótipos podem ser utilizados em várias fases dentro do processo de desenvolvimento de produtos, entre as quais se destacam a aprendizagem, comunicação, integração e marco de projeto, conforme segue: 
a) aprendizagem: os protótipos são construídos principalmente para responder questões de projeto, funcionando como uma ferramenta de aprendizagem a cada integração em que são utilizados;

b) comunicação: os protótipos desempenham o papel principal no compartilhamento de déias, atuando como catalisadores para a troca de informações num ambiente de projeto composto por pessoas com diferentes habilidades e pontos de vista. A comunicação em todos os níveis fica facilitada principalmente quando se utilizam protótipos físicos, pois a representação física de um produto é muito mais fácil de ser entendida do que um desenho técnico ou uma descrição verbal;

c) integração: os protótipos promovem e melhoram a integração entre os membros de uma organização multicultural e multifuncional, por atuarem como um meio de comunicação e entendimento comum e, também, como uma base de dados em um processo de desenvolvimento de produtos. A possibilidade de se executar montagens nos estágios iniciais do processo de desenvolvimento de produtos, aumenta o nível de integração e promove a solução de problemas de projeto;

d) marco de projeto: os protótipos podem ser utilizados como marcos no processo de desenvolvimento de produtos por meio do estabelecimento de objetivos a serem alcançados. Assim, eles possibilitam a demonstração de progresso e reforçam o uso de cronogramas. Um cronograma que prevê protótipos serve como um coordenador de atividades de subsistemas paralelos.

\subsection{Vantagens ao se utilizar protótipos no desenvolvimento de produtos}

Na utilização de protótipos na fase de desenvolvimento de produtos pode-se considerar que uma das vantagens principais está relacionada à utilização dos protótipos na certificação que uma peça complexa necessita em todas suas características.

O produto pode ser otimizado de várias formas com o uso de protótipos, uma contribuição fundamental é a possibilidade de facilitar o processo de decisão baseado em resultados de simulações, avaliações, testes e integração de clientes pilotos nas fases iniciais do projeto.

O protótipo é um veículo que permite ao marketing simular as respostas dos consumidores ou finalizar os requerimentos de um projeto. A possibilidade de obter protótipos rapidamente, favorece a otimização do produto pela possibilidade do teste de diferentes hipóteses, ou seja, explorando e avaliando soluções alternativas, assim, o processo de desenvolvimento de produtos também pode ser otimizado. 
Um protótipo técnico pode ser usado como garantia de eficiência da equipe responsável pelo desenvolvimento do produto, ele serve como uma confirmação da qualidade do produto e do processo, também possibilita uma melhor documentação do processo de desenvolvimento de produtos.

Na manufatura, os protótipos podem atuar como base para outros modelos mestres para processos de fabricação, a exemplo de modelo mestre para processos, como fundição em cera perdida ou a vácuo, entre outros. Podem também auxiliar na simulação e aprovação do processo de manufatura, possibilitando a verificação de ferramentas e dispositivos necessários.

É importante observar a influência dos estágios iniciais do processo de desenvolvimento de produtos, que compreendem a fase de projeto, no custo comprometido do produto. Os custos despendidos em avaliação nas fases iniciais darão retorno na forma de grandes economias em estágios mais avançados. É comum, no entanto, encontrar resistência contra a alocação de etapas de prototipagem no processo de desenvolvimento de produtos, argumentando que estas requerem muito tempo.

O tempo ganho com a omissão destas etapas geralmente resulta em prejuízo da qualidade do produto e mais tempo para o seu desenvolvimento. Por outro lado, o reconhecimento imediato de problemas de projeto já é responsável pela redução do tempo total de desenvolvimento de um produto. O uso de protótipo possibilita a detecção de problemas que poderiam, de outra forma, ser descobertos somente depois de finalizadas as atividades dispendiosas do processo de desenvolvimento.

O custo de alterações de projeto é maior quando em estágios mais adiantados do processo de desenvolvimento de produtos.

A seguir (tabela 1 - custos de projeto), é apresentada uma ideia de custos de mudanças de engenharia em três estágios do processo, dados fornecidos pela empresa CIMACAD, desenvolvedora de projeto e inovações em novos produtos.

Tabela 1: Custos de projeto

\begin{tabular}{|c|c|c|}
\hline \multicolumn{3}{|c|}{$\begin{array}{l}\text { Custo da Mudança de Engenharia em várias etapas do processo de desenvolvimento de } \\
\text { produtos }\end{array}$} \\
\hline Etapas do PDP & Descrição & $\begin{array}{l}\text { Custo Médio por } \\
\text { mudança }\end{array}$ \\
\hline 1 & Fase de simulação preditiva. & $R \$ 2.000,00$ \\
\hline 2 & Fase de testes, antes da liberação do produto. & $\mathrm{R} \$ 20.000,00$ \\
\hline 3 & Depois da liberação do produto. & $\mathrm{R} \$ 100.000,00$ \\
\hline
\end{tabular}


Além de diminuir a probabilidade de alterações nas fases mais adiantadas do processo, a taxa de sucesso do processo de desenvolvimento de produtos pode ser melhorada com o uso de protótipo, devido ao aumento na confiabilidade das informações para as etapas seguintes. Cada ciclo de prototipagem representa um ganho de experiência e se traduz em aprendizado para todos os envolvidos no processo de desenvolvimento de produtos, pois novas informações são obtidas, ajudando a enfocar os esforços subsequentes na direção correta para melhoria do produto.

\subsection{Materiais e métodos}

O processo de modelagem por fusão e deposição é baseado na extrusão de filamentos de plásticos aquecidos. A máquina FDM possui um cabeçote que se movimenta no plano horizontal (plano xy) e uma plataforma que se movimenta no sentindo vertical (eixo z). No cabeçote, fios de material termoplástico que são forçados, por guias rotativas, a atravessarem dois bicos extrusores aquecidos a 270 으 durante o processo de fusão.

Após a primeira etapa, um bico recebe material para a construção do objeto 3D, enquanto outro recebe material para ser utilizado como suporte para a fabricação de superfícies suspensas ou complexas.

Na sequência, o cabeçote movimenta-se no plano xy enquanto as guias rotativas empurram o fio para o interior do bico extrusor fazendo com que o material seja aquecido, extrudado e depositado.

Ao final de cada camada, a plataforma se desloca para baixo, com uma distância igual à espessura de camada $0,257 \mathrm{~mm}$, e o cabeçote começa a extrudar novos filamentos para construir uma nova camada sobre a anterior, repetindo este procedimento até formar por completo o objeto 3D. A figura 1 apresenta um esquema simplificado do processo FDM.

Figura 1: Esquema simplificados do processo FDM

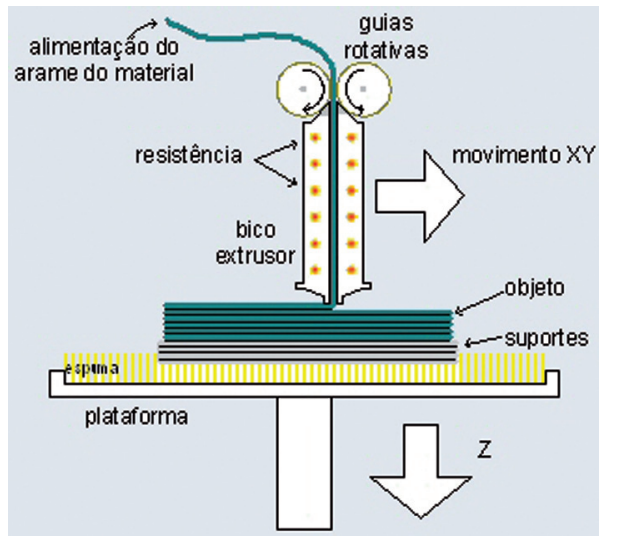

Fonte: Stratasys (2012) 
Através do processo FDM é possível fabricar objetos em ABS (acrilonitrila-butadienoestireno), PC (policarbonato), elastômero e cera. A tabela 2 apresenta alguns dos materiais comercialmente disponíveis para a fabricação de objetos pelo processo FDM, em sua maioria são fornecidos pela empresa Stratasys (E.U.A.).

Quadro 1: Principais materiais utilizados no processo FDM

\begin{tabular}{|c|c|c|}
\hline Nome comercial & Tipo & Aplicação \\
\hline Stratasys (EUA) & Filamento de $A B S$ & $\begin{array}{l}\text { Durável, resistente, boa } \\
\text { resistência térmica e química. } \\
\text { Colorido. }\end{array}$ \\
\hline ABSi $500 \quad$ Stratasys (EUA) & $\begin{array}{l}\text { Filamento de metil- } \\
\text { metacrilato de ABS }\end{array}$ & $\begin{array}{l}\text { Aplicações médicas. Poder } \\
\text { ser esterilizado com radiação } \\
\text { gama. }\end{array}$ \\
\hline IC W06 wax Stratasys (EUA) & $\begin{array}{l}\text { Filamento para modelos } \\
\text { de fundição de cera } \\
\text { perdida. }\end{array}$ & Fundição de cera perdida. \\
\hline $\begin{array}{l}\text { Elastomer E20 Stratasys } \\
\text { (EUA) }\end{array}$ & Filamento de elastômero & $\begin{array}{l}\text { Material elástico resistente e } \\
\text { flexível. Ideal para vedações e } \\
\text { peças flexíveis. }\end{array}$ \\
\hline PolycarbonateStratasys (EUA) & $\begin{array}{l}\text { Filamento de } \\
\text { policarbonato }\end{array}$ & $\begin{array}{l}\text { Termoplástico rígido de } \\
\text { performance superior ao ABS. }\end{array}$ \\
\hline $\begin{array}{l}\text { PolyphenylsulphoneStratasys } \\
\text { (EUA) }\end{array}$ & $\begin{array}{l}\text { Filamento de } \\
\text { polifenilsulfona }\end{array}$ & $\begin{array}{l}\text { Termoplástico rígido para } \\
\text { aplicações diversas. }\end{array}$ \\
\hline $\begin{array}{l}\text { Polyester P1500 Stratasys } \\
\text { (EUA) }\end{array}$ & $\begin{array}{l}\text { Waffle de polímero a base } \\
\text { de poliéster }\end{array}$ & $\begin{array}{l}\text { Utilizado em protótipos } \\
\text { conceituais }\end{array}$ \\
\hline $\begin{array}{l}\text { MultipuposeABSfilament } \\
\text { (Sibco, UK) }\end{array}$ & Filamento de ABS & $\begin{array}{l}\text { Durável, resistente, boa } \\
\text { resistência térmica, química e } \\
\text { colorido. }\end{array}$ \\
\hline
\end{tabular}

Fonte: Stratasys (2012)

\subsection{Máquina de prototipagem rápida}

A Dimension fabricada pela Stratasys gera protótipos a partir de modelos virtuais projetados em sistemas CAD 3D. O processo baseia-se no modelamento por deposição de material fundido e destaca-se pela simplicidade, por não causar danos ao meio 
ambiente e não requerer instalações complexas, podendo ser utilizada em ambiente de escritório. Desta forma, encaixes, conexões, funcionamentos e todas as interações de design podem ser imediatamente testados.

O termo prototipagem rápida ou rapidprototyping (RP) refere-se a uma classe de tecnologias que podem automaticamente construir modelos físicos a partir de um modelo gerado num sistema CAD (GORNI, 2001).

Para utilizar a Dimension, basta conectá-la à rede e carregar o softwareCatalyst, que automaticamente importa o arquivo modelado, criando uma precisa rota de deposição de material para construção do modelo. Depois basta inserir os cartuchos de material e seguir os comandos.

Múltiplas peças podem ser feitas no envelope de construção para maximizar a eficiência. O software ainda administra a fila de impressão, tempo de construção, status do material e outras informações da máquina. A Dimension funciona sem a necessidade de operador e pode fornecer informações sobre o status e término do modelo por e-mail, pager ou internet.

A impressora 3D pode ser conectada à rede TCP/IP e tem compatibilidade com estações Windows. Seu envelope de construção tem tamanho máximo em 203×203×305 mm ou 8 " $\times 8$ " $\times 12$ " e utiliza o resistente termoplástico de engenharia ABS nas cores branco, azul, amarelo, preto, vermelho e verde, além de ter configurações de cores. Figura 2 ilustra uma maquina de deposição em ABS fabricada pela StratasysDimension. Para o estudo de caso será utilizado esta máquina dentro do SENAI JOINVILLE.

Figura 2: Máquina FDM "StratasysDimension"

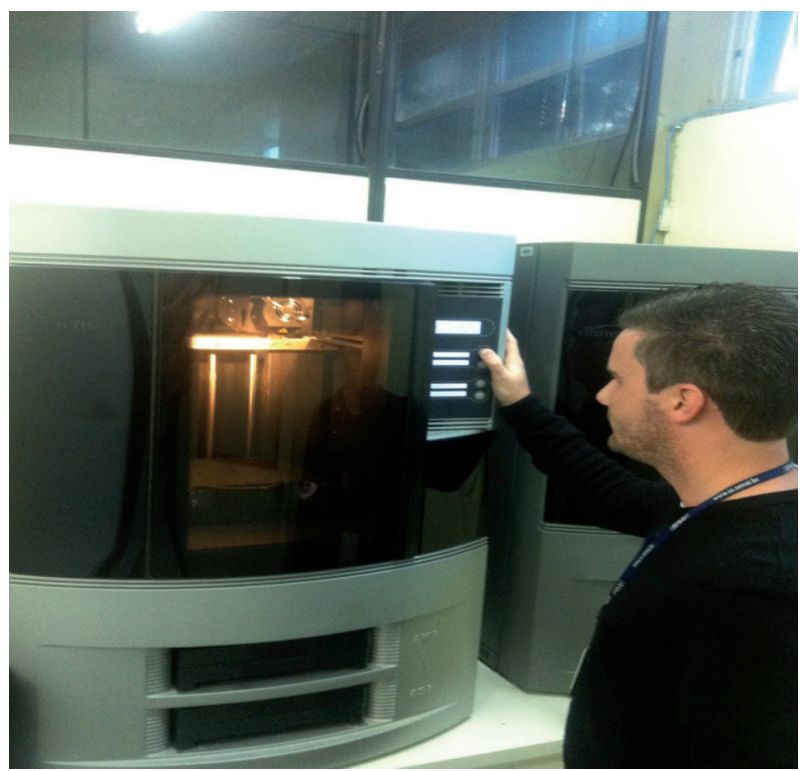

Fonte: Dos autores (2012) 


\subsection{Usinagem CNC para confecção de protótipos}

A usinagem CNC (Comando Numérico Computadorizado) é muito utilizada na fabricação de protótipos. O processo inicia-se a partir de um bloco bruto de onde a ferramenta de corte remove material até formar a peça desejada. Com a usinagem CNC pode-se fazer protótipos nos mais variados materiais.

O controle numérico é um método de controle dos movimentos de máquinas pela interpretação direta de instruções codificadas na forma de números e letras.

O sistema interpreta os dados e gera o sinal de saída que controla os componentes da máquina. Posteriormente, estas máquinas foram equipadas com controle numérico computadorizado (CNC) aumentando a flexibilidade, precisão e versatilidade. O controle numérico computadorizado é uma evolução do controle numérico, que substituiu o controle por hardware por controle por software.

Para a confecção de protótipos em máquina CNC existe a necessidade de um software CAD/CAM, onde serão gerados os programas de usinagem que formarão a peça.

O software é o meio mais moderno para a elaboração de programas CNC, tais sistemas permitem uma interpretação da geometria das peças armazenadas em arquivo gráfico criado no CAD e a geração dos programas CNC de acordo com algumas informações fornecidas pelo programador usuário do módulo CAM.

Em termos gerais, os sistemas CAD/CAM disponíveis no mercado exigem os seguintes passos para a obtenção de programas CNC:

a) preparação das superfícies a serem usinadas;

b) seleção dos parâmetros e variáveis de processo (tolerâncias, sobremetal, ferramentas, parâmetros de corte, etc.);

c) geração, automática das trajetórias das ferramentas;

d) pós-processamento dos programas, adequando ao formato do comando numérico específico.

A necessidade de desenvolvimento dessas ferramentas de programação foi motivada pela complexidade das formas criadas e a dificuldade de elaboração de programas CNC para estas geometrias. 


\subsubsection{Fresadora CNC}

CNCs são máquinas ferramenta do tipo fresadora, originadas das fresadoras convencionais, que evoluíram e foram equipadas com comando numérico, podendo realizar todas as operações possíveis de fresamento e com recurso programado. Além destas capacidades as fresadoras CNC possuem grande precisão e receptibilidade. (SIMIELLI, 1994).

Hoje com tecnologias avançadas possuem trocadores de ferramentas automáticos, avanços e rotações cada vez mais rápidos para melhor atender o mercado de trabalho. São utilizadas em usinagem de peças seriadas ou não, sendo que sua aplicação não é indicada para altas produções, devido seu alto custo/hora. A figura 3 ilustra uma fresadora CNC.

Figura 3: Fresadora CNC - DISCOVERY 540

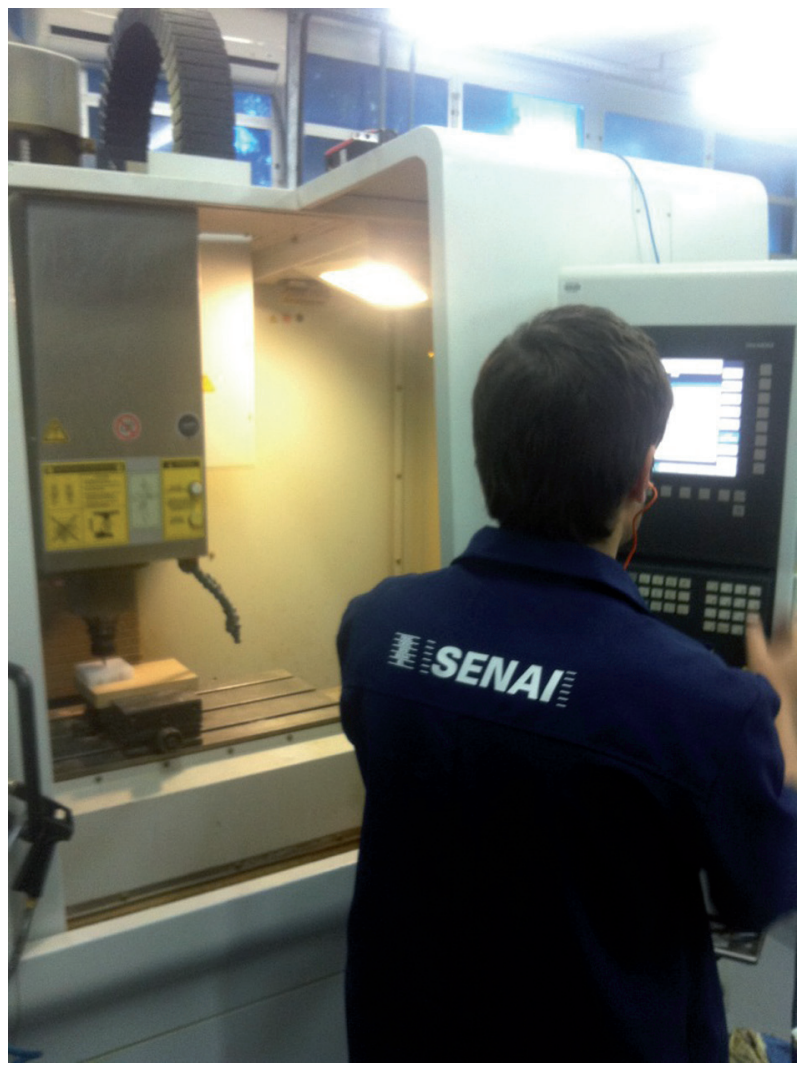

Fonte: Dos autores (2012) 


\section{ESTUDO DE CASO}

O estudo de caso foi realizado em duas etapas, na primeira foi confeccionada uma peça de geometria pré-definida para verificar as vantagens e desvantagens dos dois processos. Foi confeccionada uma peça por deposição em ABS, com parâmetros de construção padrão (espessura de camada $=0,254 \mathrm{~mm}$ e largura do filete $=0,508 \mathrm{~mm}$ ) sem acabamento nas superfícies.

A mesma peça foi confeccionada na fresadora CNC partindo de um bloco bruto com parâmetros conforme tabelas 3,4 e 5 padrões de desbaste e acabamento a seguir nos resultados desse artigo. Foram utilizadas para esta usinagem uma fresa de topo reto, de $8 \mathrm{~mm}$, uma fresa esférica de $3 \mathrm{~mm}$, uma fresa de topo de 1,5 $\mathrm{mm}$ e uma fresa de topo $4 \mathrm{~mm}$.

No acabamento foi utilizada, uma altura de passada vertical e lateral de $0,25 \mathrm{~mm}$. A figura 4 mostra uma imagem da peça usinada e, ao lado direito, a peça construída por deposição em ABS.

Figura 4: Peças utilizadas no estudo de caso 1

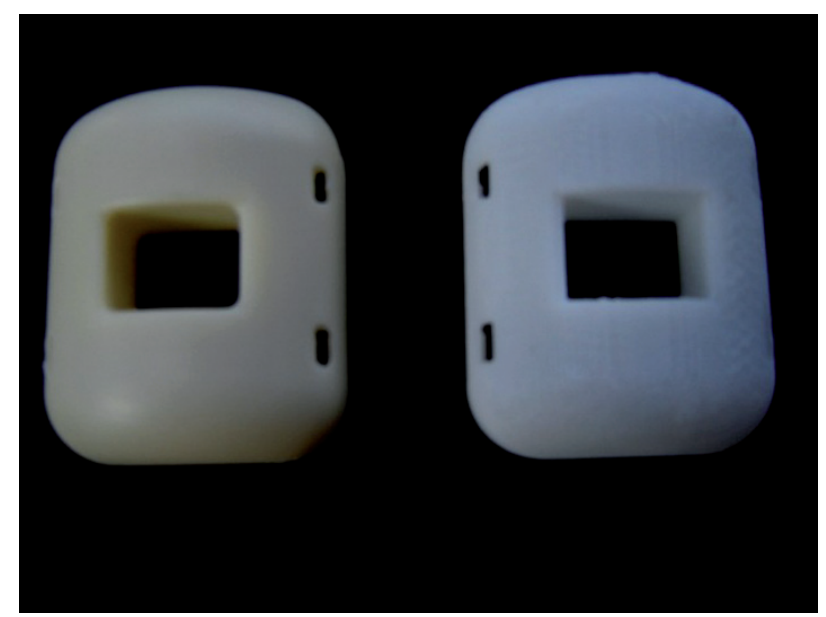

Fonte: Dos autores (2012)

No segundo estudo de caso, foram confeccionados dez corpos de prova de tração, segundo a norma ASTM D638. Os parâmetros de confecção das peças em deposição utilizadas foram: espessura de camada $=0,254 \mathrm{~mm}$ e largura do filete $0,508 \mathrm{~mm}$. Na usinagem foi utilizado um bloco na espessura certa e recortados os perfis do corpo de prova com uma passada vertical de $0,5 \mathrm{~mm}$. A figura 5 ilustra os corpos de prova que foram confeccionados, corpos de provas amarelos foram os confeccionados na deposição e os corpos brancos, usinados. 
Figura 5: Corpos de Prova

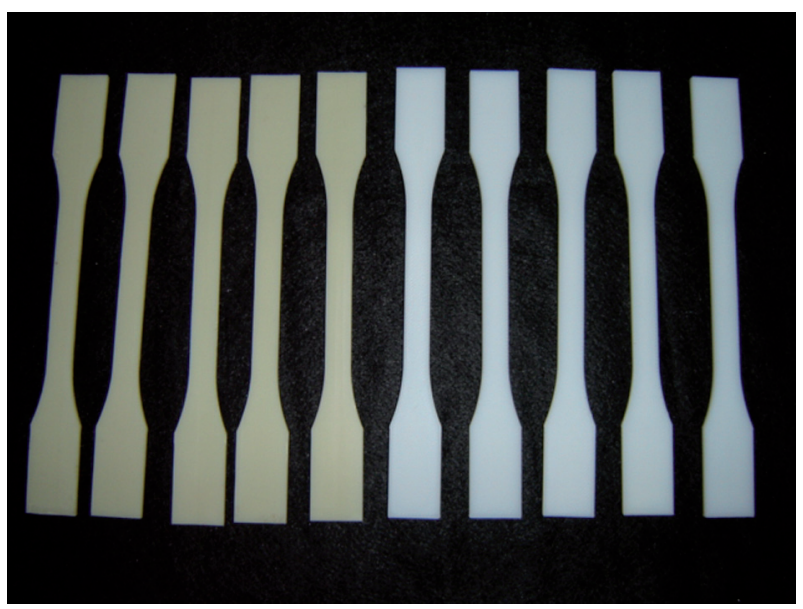

Fonte: Dos autores (2012

Os corpos de prova foram submetidos a ensaios de tração com a finalidade de dimensionar a diferença de resistência dos materiais dos dois processos de confecção. Foi utilizada uma máquina universal de ensaios INSTRON. A figura 6 exemplifica os dez corpos de prova rompidos que foram tracionados. Na esquerda os usinados e na direita os confeccionados na deposição.

Figura 6: Corpos de prova tracionados

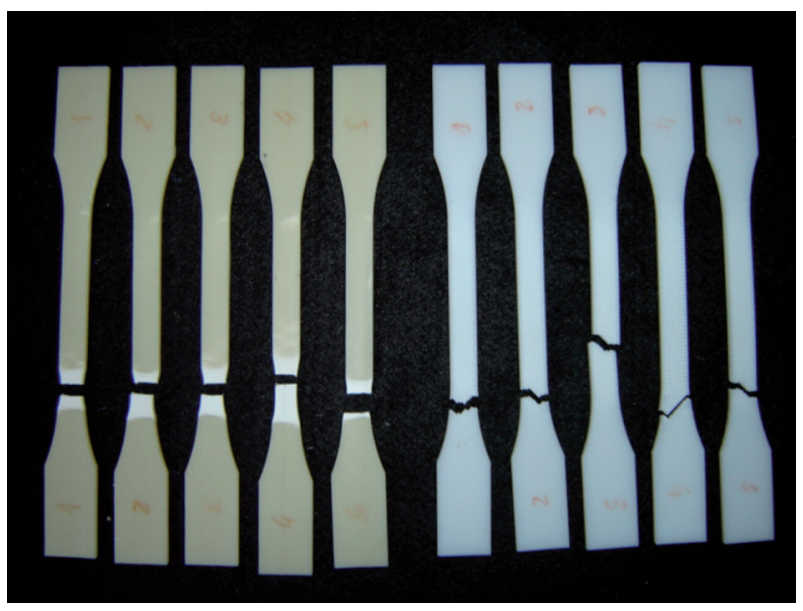

Fonte: Dos autores (2012)

Para o levantamento de custos de fabricação dos protótipos foram considerados os seguintes valores de hora/máquina e os preços dos materiais: 
a) máquina $\mathrm{CNC}=\mathrm{R} \$ 50,00$ por hora;

b) dimension $\mathrm{FDM}=\mathrm{R} \$ 45,00$ por hora;

c) chapa de $A B S 1000 \times 500 \times 30=R \$ 1300,00$;

d) chapa de ABS $1000 \times 500 \times 50=R \$ 1500,00$;

e) cartucho FDM ABS e SUPORTE = R\$2000,00;

f) programação $=\mathrm{R} \$ 40,00$ por hora;

g) espuma $=R \$ 15,00$ peça.

Com estes dados foram levantados os custos das peças confeccionadas e realizou-se uma comparação para dimensionar o processo com menor custo.

\section{RESULTADOS}

No primeiro estudo de caso foi possível identificar as vantagens e desvantagens da peça confeccionada em deposição e da usinada. Também foi realizada uma comparação de tempo e custo de confecção entre os dois processos. A peça em deposição apresentou na sua superfície raiada, marcas relativas ao espaçamento entre camadas e filetes conforme mostra a figura 7.

Figura 7: Protótipo

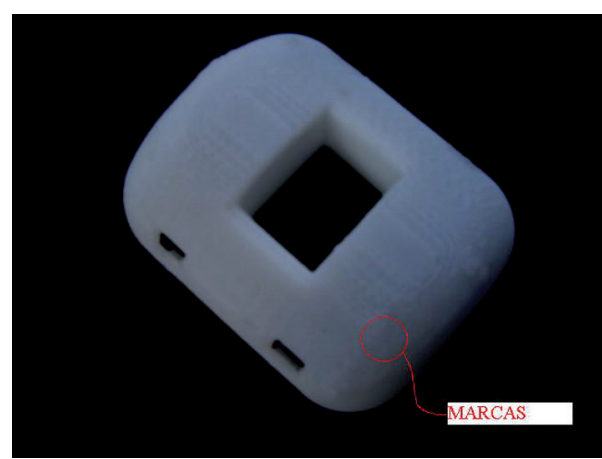

Fonte: Dos autores (2012)

Já na peça usinada, nesta mesma área observou-se um melhor acabamento como mostra a figura 8. Isto porque o acabamento da peça usinada é feito em três eixos. Neste processo é mais prático dar acabamento da superfície. 
Figura 8: Protótipo Usinado

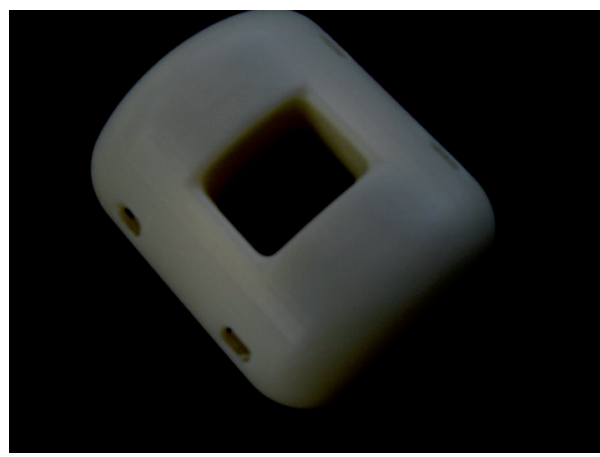

Fonte: Dos autores (2012)

Na peça usinada, observa-se raios onde deveriam existir cantos vivos. Isto ocorreu devido à usinagem ser utilizada uma fresa, como pode ser visto na figura 9 - item (A). A peça em deposição ficou com cantos vivos sem problemas como mostra a figura 9 - item (B). Para este problema da peça usinada, já foram criados procedimentos posteriores para quebrar os raios que ficam na usinagem CNC.

Figura 9: Item (A e B)

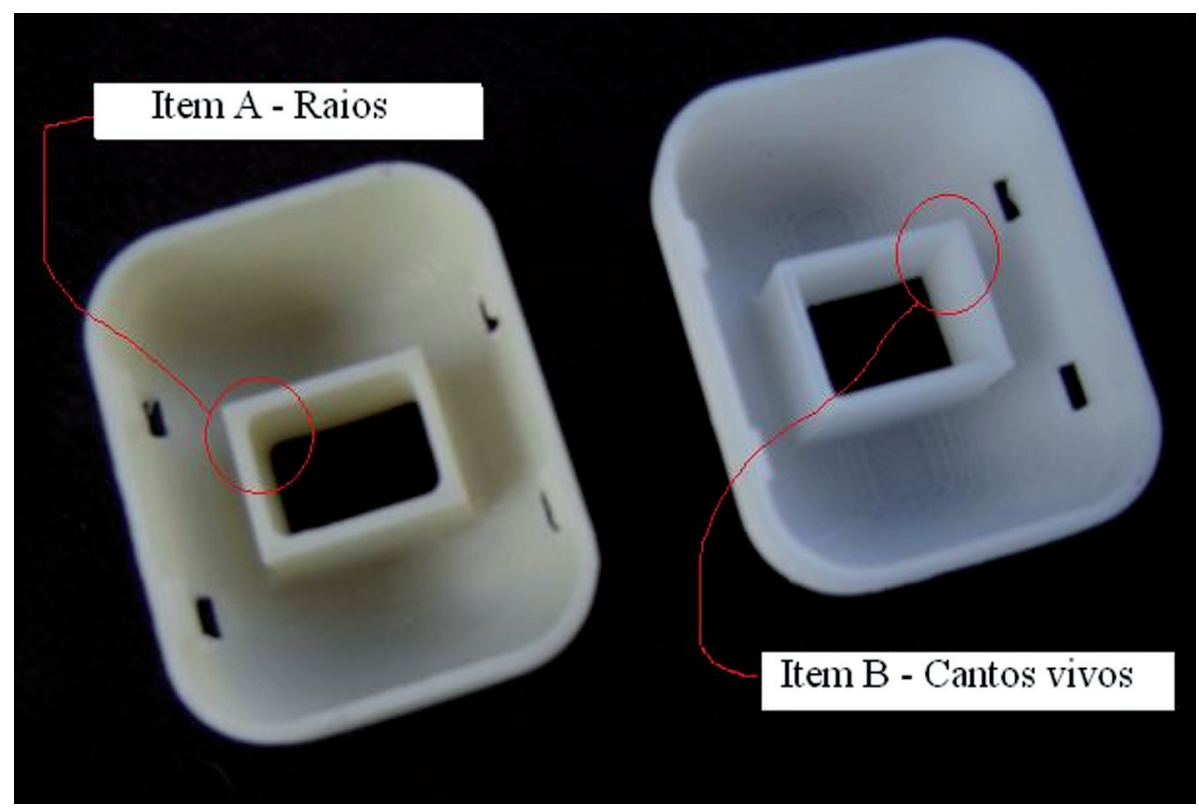

Fonte: Dos autores (2012) 
Para a peça usinada houve a necessidade de utilizar uma "cama" de poliuretano expandido para encaixar o primeiro lado da peça e possibilitar a usinagem do segundo lado. Neste processo utilizou-se um adesivo após o término, foi necessário raspar o excesso de cola que fica na peça.

O processo de deposição também utiliza um material chamado suporte, que serve para manter a peça presa a base de espuma após a construção o mesmo deve ser extraído da peça. O custo das peças prototipadas em cada processo varia de geometria para geometria, nesse caso específico foi realizada uma análise de tempos e materiais gastos, definindo o valor do custo de cada peça.

Também foram criadas três situações de custos de produção para a mesma peça, partindo com 10 gramas, depois com 37 gramas e por último com 83 gramas. Foram levantados os custos de produção das peças prototipadas nos dois processos. A peça de 10 gramas tem dimensões externas de $50 \times 40 \times 2(\mathrm{~mm})$ e esta representada na tabela 2 .

Tabela 2: Levantamento de custo da peça 1prototipada

\begin{tabular}{l|l|l|l|l}
\hline PEÇA 1 & OPERAÇÃO & QUANTIDADE & VALOR/PEÇA(R\$) & TOTAL(R\$) \\
\hline PÇ USINADA & PROGRAMAÇÃO & $60 \mathrm{~min}$ & $40,00(60 \mathrm{~min})$ & 40,00 \\
& USINAGEM & $75 \mathrm{~min}$ & $50,00(60 \mathrm{~min})$ & 62,50 \\
\hline TOTAL & MATERIAL & $1 / 250$ & 1300,00 (chapa) & 5,20 \\
\hline PEÇA FDM & ESPUMA & & & $R \$ 107,7$ \\
\hline & PROGRAMAÇÃO & $10 \mathrm{~min}$ & $40,00(60 \mathrm{~min})$ & 6,66 \\
\hline TOTAL & TEMPO & $75 \mathrm{~min}$ & $45,00(60 \mathrm{~min})$ & 56,25 \\
\hline
\end{tabular}

Fonte: Dos autores (2012)

Após os resultados, foram aumentadas as dimensões das peças para $75 \times 60 \times 30 \mathrm{~mm}$, gastando 37 gramas de material na FDM. Após realizou-se uma segunda análise de custos que mostra a tabela 3. 
Tabela 3: Levantamento de custo da peça 2 prototipada.

\begin{tabular}{l|l|l|l|l}
\hline PEÇA 2 & OPERAÇÃO & QUANTIDADE & VALOR/PEÇA(R\$) & TOTAL(R\$) \\
\hline PEÇA USINADA & PROGRAMAÇÃO & $90 \mathrm{~min}$ & $40,00(60 \mathrm{~min})$ & 60,00 \\
& USINAGEM & $112,5 \mathrm{~min}$ & $50,00(60 \mathrm{~min})$ & 93,75 \\
\hline TOTAL & MATERIAL & $1 / 110$ & 1300,00 (chapa) & 11,81 \\
\hline PEÇA FDM & ESPUMA & $1 / 6 \mathrm{pc}$ & 15,00 (peças) & 2,50 \\
\hline & PROGRAMAÇÃO & $10 \mathrm{~min}$ & $40,00(60 \mathrm{~min})$ & 6,66 \\
\hline TOTAL & TEMPO & $188,4 \mathrm{~min}$ & $45,00(60 \mathrm{~min})$ & 141,3 \\
\hline
\end{tabular}

Fonte: Dos autores (2012)

O resultado da tabela 4 proporcionou uma grande surpresa, pois foi aumentada a peça para $100 \times 80 \times 40 \mathrm{~mm}$, utilizada 83 gramas de material na FDM e confirmamos o resultado da tabela anterior que nos mostra que com o aumento da peça a FDM obtém um valor mais elevado, vide tabela 4.

Tabela 4: Levantamento de custo da peça 3 prototipada

\begin{tabular}{|l|l|l|l|l}
\hline PEÇA 3 & OPERAÇÃO & QUANTIDADE & VALOR/PEÇA (R\$) & TOTAL(R\$) \\
\hline PEÇA USINADA & PROGRAMAÇÃO & $120 \mathrm{~min}$ & 40,00 (60min) & 80,00 \\
& USINAGEM & $150 \mathrm{~min}$ & 50,00 (60min) & 125,00 \\
\hline TOTAL & MATERIAL & $1 / 62,5$ & 1500,00 (chapa) & 24,00 \\
\hline PEÇA FDM & ESPUMA & $1 / 4 \mathrm{pc}$ & 15,00 (peças) & 3,75 \\
\hline & PROGRAMAÇÃOO & $10 \mathrm{~min}$ & $40,00(60 \mathrm{~min})$ & 6,66 \\
\hline TOTAL & TEMPO & $373,2 \mathrm{~min}$ & $45,00(60 \mathrm{~min})$ & 279,9 \\
\hline
\end{tabular}

Fonte: Dos autores (2012) 
As figura 10 a 11, mostram os custos relacionados nas tabelas 3,4 e 5 .

Figura 10: Comparação de custos

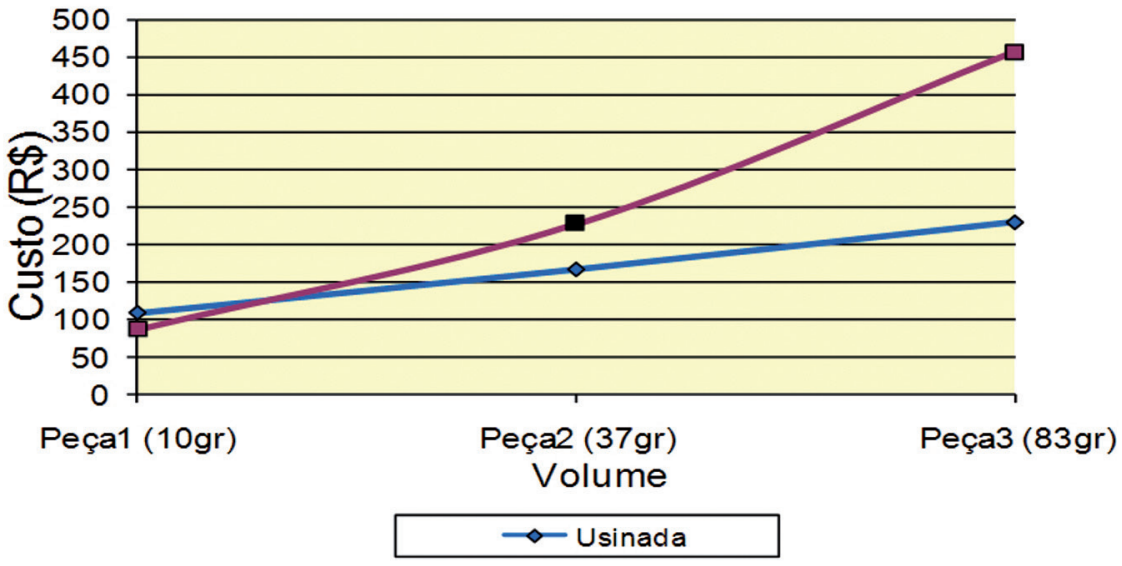

Fonte: Dos autores (2012)

No gráfico volume $x$ custos, pode-se identificar que na primeira peça os dois processos estão próximos, mas com o aumento do volume, obtém-se uma curva muito acentuada para a FDM, enquanto a usinagem cresce linearmente.

Figura 11: Volume $x$ tempo

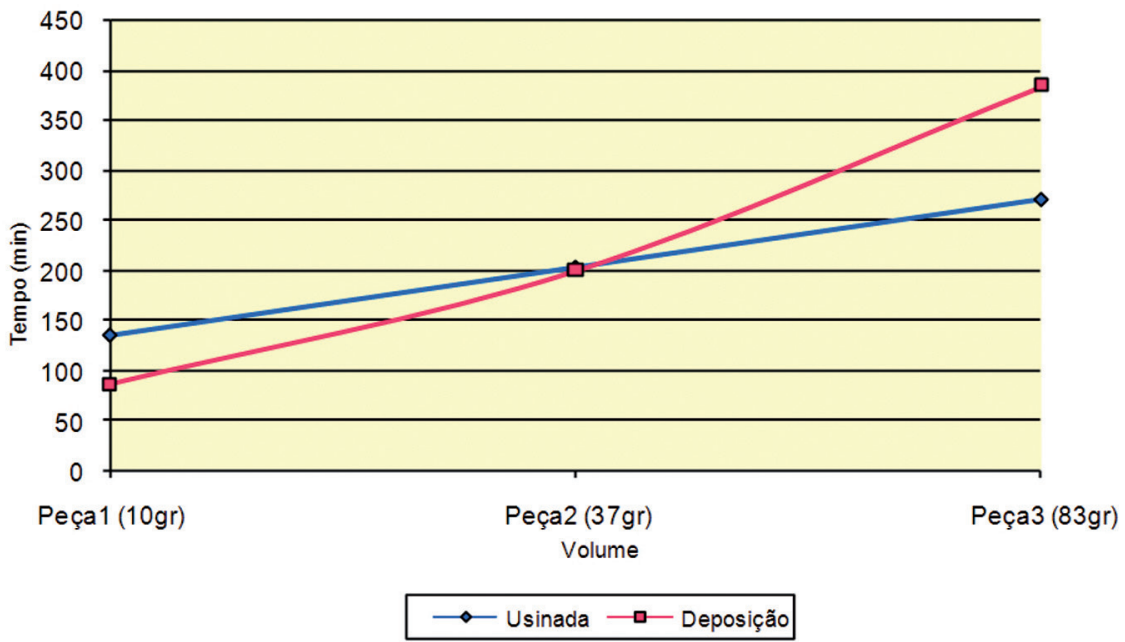

Fonte: Dos autores (2012)

No gráfico volume $x$ tempo, foi obtido no resultado uma mínima diferença entre volume $x$ custo, pois a proximidade aconteceu na segunda peça, já na terceira peça, a FDM 
novamente se tornou muito mais cara. Na figura 12,13 e 14 foram feitos um comparativo entre os passos que são utilizados nos dois processos. A programação, o tempo de execução e a material.

Figura 12: Comparativo de custo peça 1

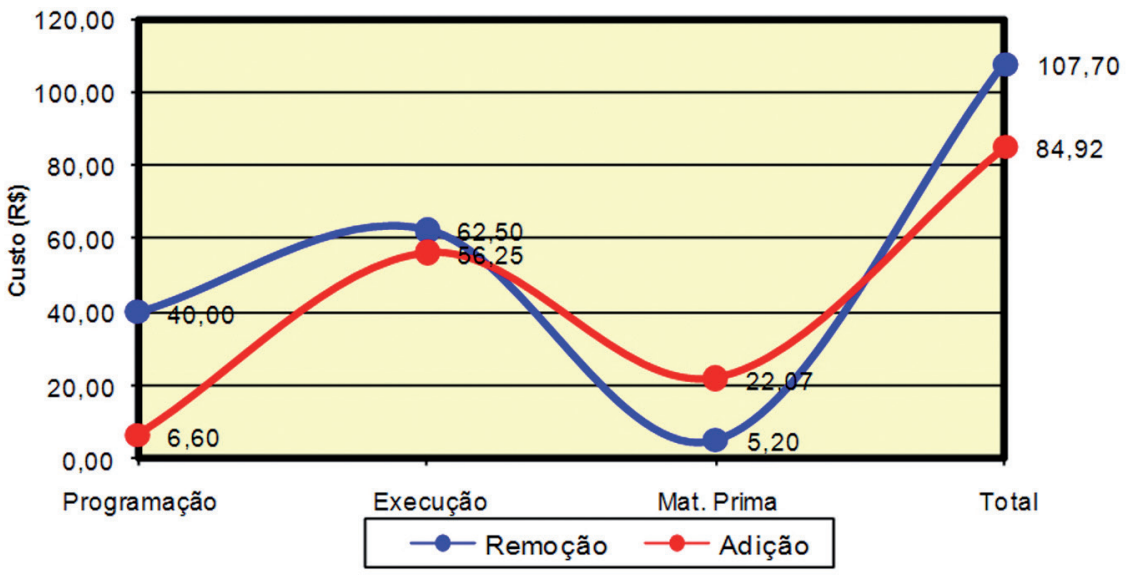

Fonte: Dos autores (2012)

Figura 13: Comparativo de custo peça 2

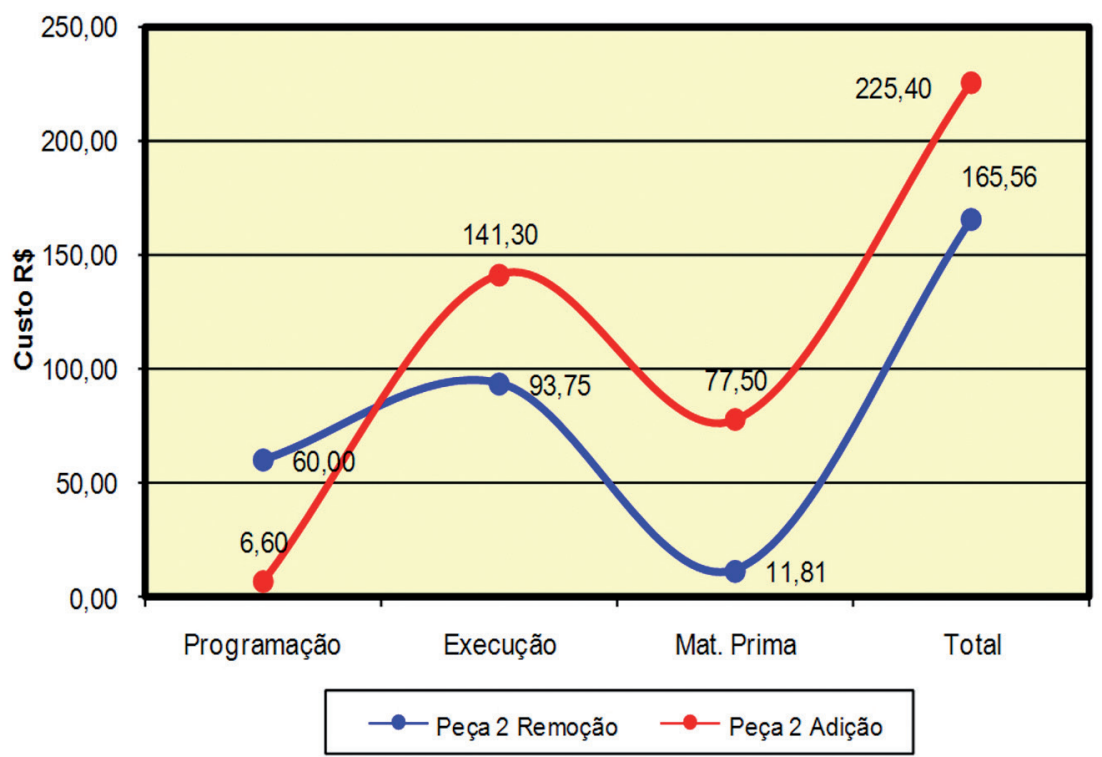

Fonte: Dos autores (2012) 
Figura 14: Comparativo de custo peça 3

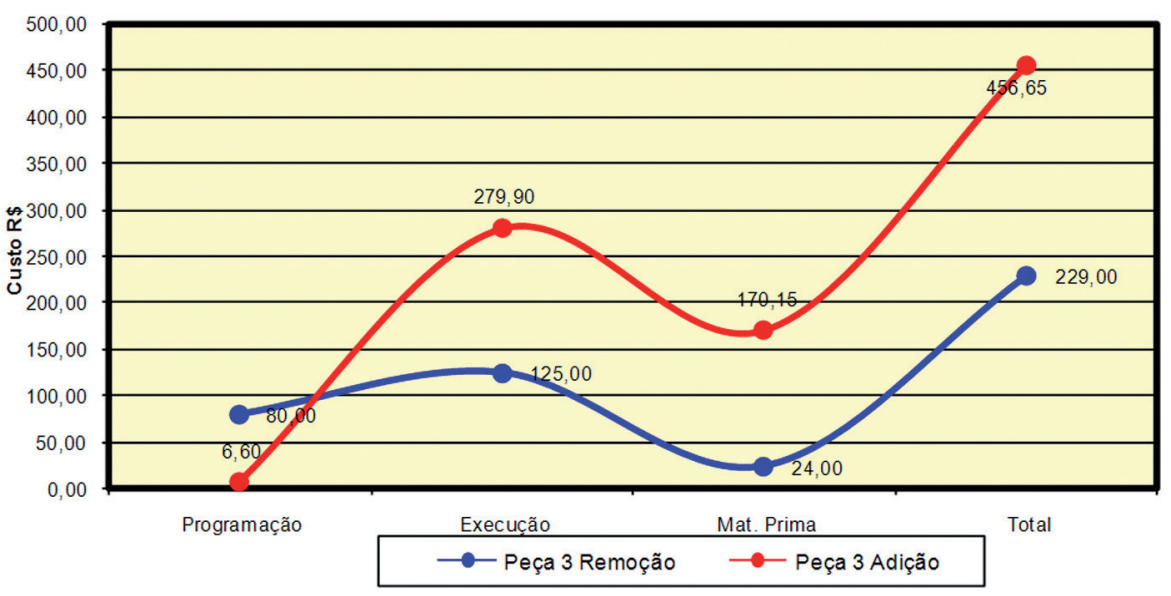

Fonte: Dos autores (2012)

Estes comparativos mostram que independente da peça confeccionada, a FDM sempre terá uma programação mais barata e rápida. Já o material da usinagem sempre será mais barato que o da FDM. Porém, o tempo de execução, já é variável, e depende muito do formato e tamanho da peça.

É possível observar que, com o aumento de tamanho da peça, a usinagem torna-se o processo mais vantajoso, mas a usinagem CNC não consegue produzir a peça com maior velocidade que o processo de deposição.

No segundo estudo de caso, foi realizado ensaio de tração para investigar as propriedades mecânicas dos dois materiais após a confecção das peças.

Tabela 5: Resultados dos ensaios de tração

\begin{tabular}{l|l|l}
\hline \multirow{2}{*}{ Corpo de provas em ABS } & Norma ASTM & Resistência à Tração \\
& D-638 & $\left(\mathbf{k g f} / \mathbf{c m}^{\mathbf{2}}\right)$ \\
Peça Usinada & Valor médio & 331,87 \\
& Desvio padrão & 3,84 \\
\hline Peça FDM & Valor médio & 123,38 \\
& Desvio padrão & 3,21 \\
\hline
\end{tabular}

Fonte: Dos autores (2012) 
O valor especificado pelo fornecedor do $A B S$ indicava uma resistência a tração de $450 \mathrm{kgf} / \mathrm{cm}^{2}$ para a chapa usinada e $220 \mathrm{kgf} / \mathrm{cm}^{2}$ para o ABS da FDM. Os ensaios de tração mostraram que a peça usinada é três vezes mais resistente que a depositada. Foi identificado que os corpos de provas em FDM rompem-se com facilidade no formato dos filamentos depositados e não chegam nem a sofrer escoamento.

\section{CONCLUSÃO}

Após a realização do estudo de caso e análise dos resultados, é possível concluir que ambos os processos tem seus pontos fortes e fracos. Sendo possível definir qual será o mais barato, sem conhecer a peça que será confeccionada.

Pode-se identificar com o levantamento dos custos, que para alguns protótipos é mais viável realizar o protótipo usinado ao invés do protótipo por deposição, devido ao preço e tempo gasto. Por exemplo, os corpos de provas usinados levaram 130 minutos para serem confeccionado (cinco peças), e na deposição em ABS, utilizou-se 31 minutos para confeccionar cada um num total de 155 minutos. Já no estudo de caso 1, e 85 minutos para serem confeccionados na deposição e 135 minutos na usinagem CNC, tornando assim, o processo de deposição mais rápido.

Foram aumentadas ainda as dimensões das peças e identificada uma curva acentuada quando se trata de peças maiores, tornando a usinagem CNC mais em viável devido ao tempo de confecção ser menor e o material mais barato.

É possível ainda concluir que no caso de peças com raios grandes deve-se utilizar os protótipos usinados para obter o melhor acabamento. Já, para peças com muitos cantos vivos deve-se utilizar a deposição em ABS, eliminando o retrabalho manual de quebra de cantos.

Existem ainda procedimentos para melhorar o acabamento da peça na deposição com grande facilidade e com ferramentas apropriadas. Assim, é possível criar os cantos vivos com precisão nas peças usinadas. Então, pode-se entender que para o acabamento os dois processos têm-se desvantagens, que não impactam diretamente na escolha do melhor processo.

Os ensaios de tração mostraram que existe uma diferença de resistência elevada entre os dois tipos de protótipos. Assim, define-se o protótipo confeccionado a necessitar de uma maior resistência. Deve-se optar pelo protótipo usinado, entretanto, mesmo com o índice menor de resistência do protótipo produzido em deposição, os resultados foram excelentes. Isto porque as peças protótipos, em muitos casos, são utilizadas apenas para aprovação de projetos ou uma simples montagem funcional. Desta forma, com a resistência encontrada, dificilmente o protótipo se quebrará no caso de uma queda acidental, ou numa montagem simples que necessite um pouco de resistência. Esses são os maiores problemas dos materiais de prototipagem rápida. 
Os resultados dos estudos de caso serviram como referência para ambos os processos e demonstraram que é viável investir em prototipagem rápida, mas também investir em máquina CNC e, não substituí-las. Também serviu como informação sobre a resistência dos protótipos confeccionados, pois não havia nada catalogado sobre esse assunto até o momento.

\title{
PROTOTYPES FOR CONSTRUCTION OF ADDITION AND REMOVAL OF MATERIAL DEPOSITION IN COMPARISON BETWEEN ABS X CNC MACHINING
}

\begin{abstract}
This article was designed to draw a comparison between the manufacture of prototypes with two different manufacturing technologies: ABS deposition and CNC machining. In recent years prototyping has been growing in scale with the development of new technologies focusing on the care and needs required by the metal-mechanic sector. With the development of these technologies a range of options for developing their prototyped models have gained customers. With the help of two case studies, it was possible to identify and analyze the main characteristics of two prototype manufacturing processes, deposition and material removal. Both using as raw materials the ABS, identifying its advantages and disadvantages in both cases. We compared two parts with pre-defined geometry, where in the first case (deposition material) problems related to the surface finish of the prototype were identified, while in the second case (CNC) results relating to the strength mechanics were identified, after tensile tests on a series of specimens were performed by laboratory technicians at the laboratories of Senai Joinville. After performing the comparative assessment of costs, the deposition process was found to have a lower cost for smaller parts, but with increasing mass, the CNC machining process achieved a lower cost mainly due to time and material expense.
\end{abstract}

Keywords: Prototyping. CNC machining. Deposition of material. 


\section{REFERÊNCIAS}

BAXTER, M. Projeto de produto. São Paulo, SP: Edgard Blücher, 1998.

GORNI, A. Augusto Introdução a Prototipagem Rápida e Seus Processos. Revista Plástico Industrial, março (2001), p. 230-239.

LIMA, C.B.S. Engenharia Reversa e Prototipagem Rápida: estudo de caso. 2009. 91 f. Dissertação (Mestrado Engenharia Mecânica) - Universidade Estadual de Campinas, Campinas, 2009.

SIMIELLI, Edson R. Termoplástico de Engenharia. São Carlos: ABPol, 1994.

STRATASYS. FDM - Fused Deposition Modeling. [S.I.: s.n.], 2012. Disponível em: <http:// www.cimject.ufsc.br/knowledge/06_knowledge_FDM.htm>. Acesso em: 03 set. 2012.

TOLEDO, E. M.; BRITO, E. P. Z. O desenvolvimento de produtos a partir de novas tecnologias. In: EnANPAD, 23., 1999, Foz do Iguaçu, PR. Anais... Foz do Iguaçu: ANPAD, 1999. p.1-15.

VOLPATO, Neri (Edit.). Prototipagem rápida: tecnologias e aplicações. São Paulo, SP: Edgard Blücher, c2007.

\section{SOBRE OS AUTORES}

\begin{tabular}{|c|c|}
\hline & $\begin{array}{l}\text { Tecnólogo em Gestão da Qualidade e Produtividade graduado pela SOCIESC } \\
\text { e Pós Graduando do curso de Pós Graduação em Engenharia da Manutenção } \\
\text { Mecânica pelo SENAI Joinville. Técnico em Ferramentaria de Moldes e Técnico } \\
\text { em Mecânica Geral. É especialista em metal mecânico pelo SENAI Joinville. }\end{array}$ \\
Alexandre de & $\begin{array}{l}\text { Atuou como coordenador de PCP por sete anos em uma grande ferramentaria } \\
\text { situada na região de Joinville/SC, instrutor e consultor do SENAI SC em Joinville/ } \\
\text { SC para consultorias em usinagem técnica e prototipagem rápida. Foi docente } \\
\text { do curso de Ferramentaria de Moldes em processo de usinagem. É o responsável } \\
\text { pelo setor de usinagem de protótipos do SENAI Joinville. }\end{array}$ \\
\hline
\end{tabular}




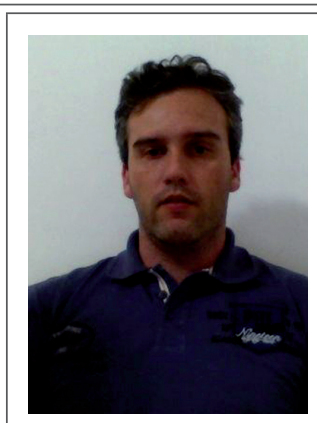

Pós Graduado em Gerenciamento de Projetos, Tecnólogo em Fabricação Mecânica pela SOCIESC. Atua no ramo de prototipagem a mais de dez anos com experiência em usinagem CNC, prototipagem por deposição de material, Engenharia reversa, modelamento e desenvolvimento de produtos, projetos de moldes de injeção. Representa o software Cimatron com a empresa CIMACAD.

Cesar Augusto

Guerra

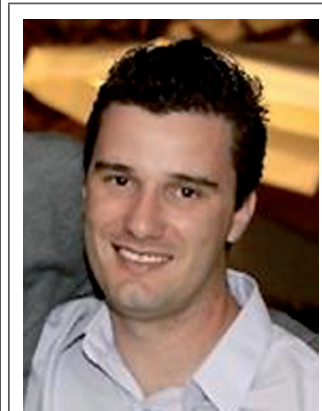

Tecnólogo em Fabricação Mecânica pelo Serviço Nacional de Aprendizagem Industrial (SENAI). Atua no ramo de prototipagem há nove anos com experiência em usinagem CNC, prototipagem por deposição de material, Engenharia reversa, modelamento e desenvolvimento de produtos, projetos de moldes de injeção. É responsável pelo Laboratório de prototipagem e Engenharia Reversa, do SENAI de Joinville/SC.

\section{Tiago Carlos}

Guerra 